\title{
Single-Flux-Quantum Circuits for Spiking Neuron Devices
}

\author{
Tetsuya HIROSE(YA), Ken UENO, Tetsuya ASAI, and Yoshihito AMEMIYA \\ Hokkaido University, Sapporo 060-0814, Japan \\ E-mail: hirose@sapiens-ei.eng.hokudai.ac.jp
}

Introduction: Single-flux-quantum (SFQ) circuits can be used for making spiking neuron devices, which are useful elements for constructing intelligent, brain-like computers. The device we propose is based on the integrate-andfire neuron (IFN) model and uses a SFQ pulse as an action signal or a spike of neurons. It can operate with a short delay of 100 ps or less and is probably the highest-speed neuron device ever reported.

Circuit Configuration: Our neuron device consists of three subcircuits, i.e., an input subcircuit, a leaky integrator subcircuit, and an output subcircuit as shown in Fig. 1; they correspond to dendrites, soma, and axon of neurons, respectively. The input subcircuit collects SFQ pulses from other neuron devices and transmits the pulses to the leaky integrator. The leaky integrator performs a nonlinear, threshold processing; it produces an output SFQ pulse if the total input exceeds a certain threshold. The output pulse is taken over by the output connection and is delivered to other neuron devices. The input and output connections consist of ordinary Josephson transmission lines combined with confluence buffers and splitters.

The key component of our neuron device is the leaky integrator subcircuit, consisting of Josephson memory loops from LA1 to LA5 connected with a damping resistor R1. It accepts SFQ pulses and stores the flux quanta in its loops; the stored flux quanta fade away with a certain time constant because of the damping resistor R1. If many SFQ pulses arrive in a short time, the number of the stored flux quanta reaches the threshold, and the leaky integrator produces an output pulse through the coupled inductor LB1. Immediately after the output, the integrator extinguishes

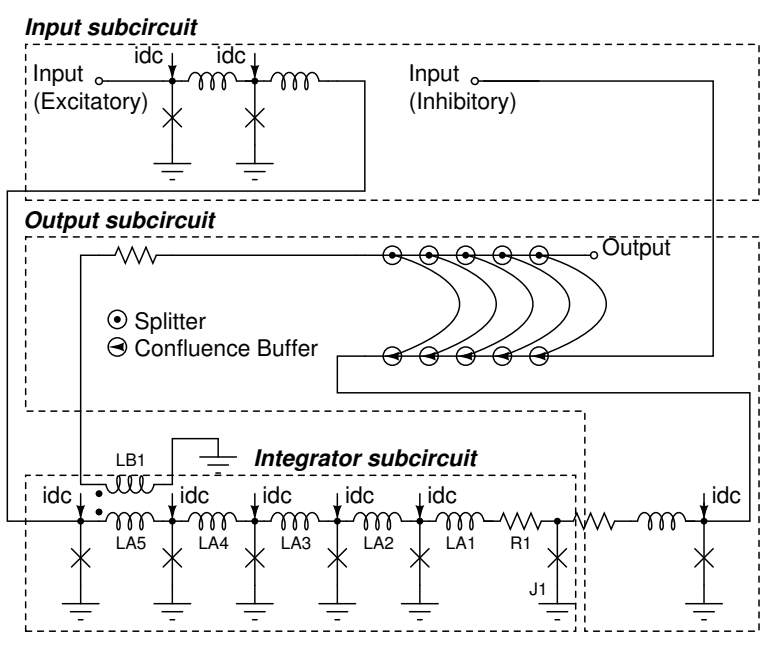

Fig. 1: Spiking neuron device consisting of singleflux-quantum circuits. idc represent a bias current. The critical current of the Josephson junction $\mathrm{J} 1$ is greater than that of other junctions. LB1 and LB5 are coupled with each other. all the stored flux quanta to initialize its state. This way our device can imitate the operation of neurons.

Results: Figure 2 shows the simulated results of the neuron device, i.e., the waveforms of (i) input pulses, (ii) the internal state, and (iii) the output of the device. The input and the output waveforms represent the voltage of the corresponding nodes of the device circuit, and the internal state shows the amount of the total current in the memory loops of the leaky integrator. For a low density of input pulses (region (A) in the figure), the device produced no output because the internal state of the device was unable to reach the threshold for firing. For a high density of the input pulses (region (B)), the internal state increased to reach the threshold, and the device produced an output pulse and then initialized its internal state (region (C)).

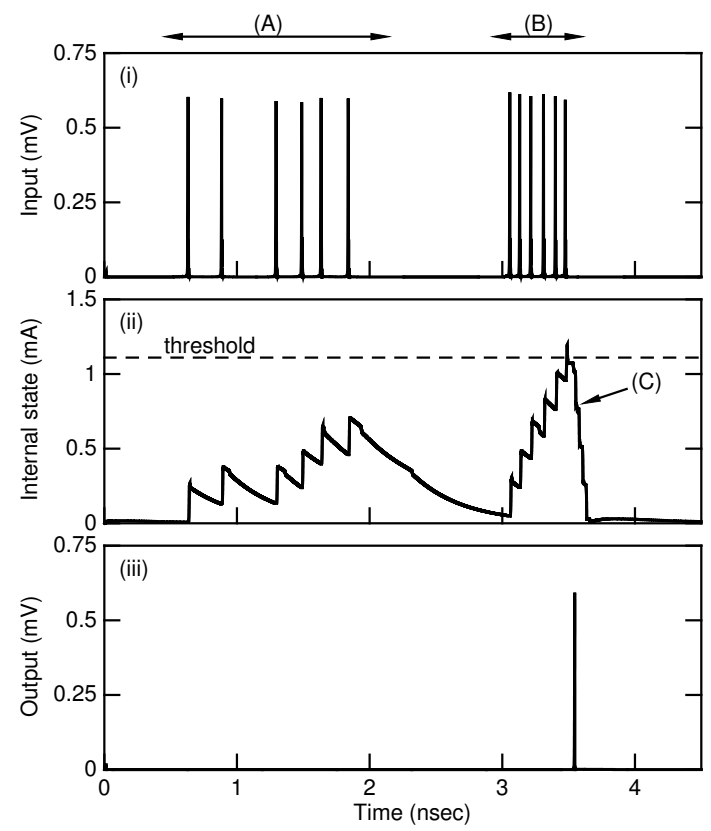

Fig. 2: Operation of the neuron device. Simulated results of (i) input pulses, (ii) internal state, and (iii) output pulse. If many input pulses arrive at short intervals, the neuron device increases its internal state and produces an output pulse.

\section{References}

[1] T.V. Duzer, et.al., "Principles of Superconductive Devices and Circuits", Prentice Hall PTR., 1999.

[2] W. Gerstner, et.al., "Spiking Neuron Models", Cambridge University Press, 2002. 